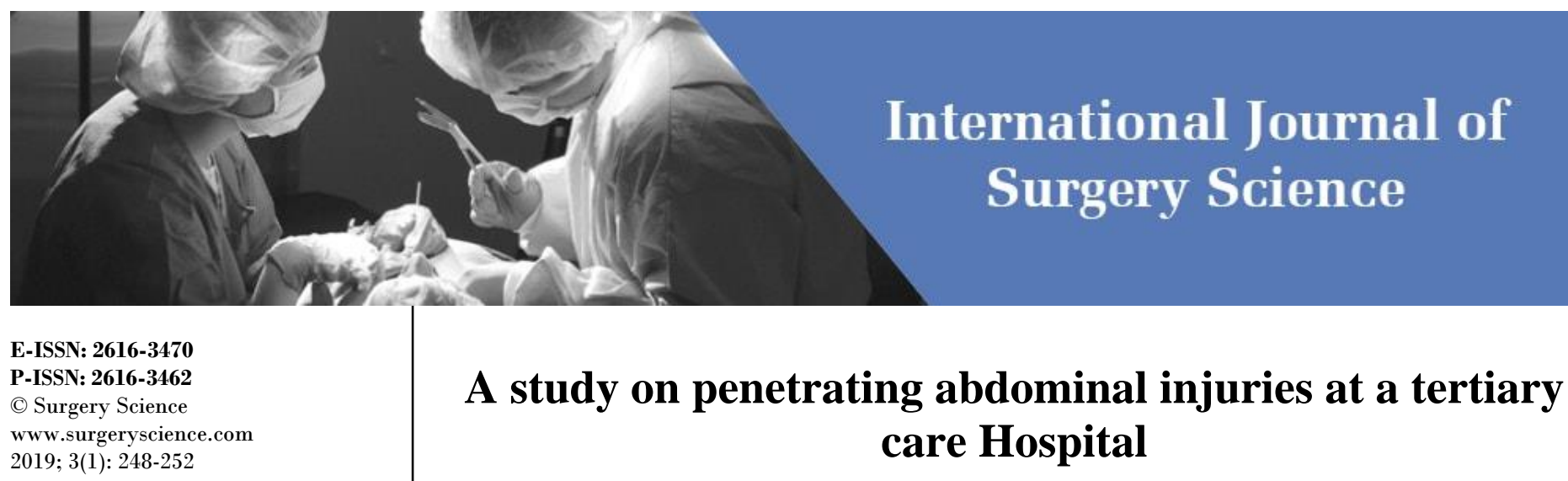

Received: 01-11-2018

Accepted: 05-12-2018

\section{Dr. Venugopala}

Associate Professor, Department of General Surgery, KIMS, Koppal,

Karnataka, India

Dr. Sunil Kumar Venkatappa Assistant Professor, Department of General Surgery, BMCRI, Bangalore, Karnataka, India
Correspondence

Dr. Sunil Kumar Venkatappa

Assistant Professor, Department of

General Surgery, BMCRI,

Bangalore, Karnataka, India

\section{Dr. Venugopala and Dr. Sunil Kumar Venkatappa}

DOI: https://doi.org/10.33545/surgery.2019.v3.i1e.41

\section{Abstract}

Evaluation of patient with abdominal trauma can be most challenging task that a surgeon to be called upon to deal with. Penetrating abdominal injuries may be parietal or visceral or perforating through and through injuries. Visceral injuries may be intraperitoneal or retroperitoneal. Stomach, duodenum, small bowel, large bowel are the organs included in the study. Once the patient is admitted the name, age, sex and mode of injury are noted. The time interval between injury and admission and time interval between admission and surgery are recorded. After retesting the patient and if time permits necessary investigation carried out. In those who are operated, the operative findings and methods of management are recorded. Cases are followed up till their discharge from the hospital. Stab injury is the common penetrating trauma accounting $64 \%$. Therewere 12 cases of bullogre injury. 4 cases of RTA, 2 cases due to falling on iron rods in a concrete centering work site (accidental fall).

Keywords: Penetrating abdominal injuries, operative findings, management

\section{Introduction}

Trauma ranks along with cardiovascular and malignancy as a major cause of morbidity and mortality. Still trauma continues to be a leading cause of death in the first four decades of life. There are various predisposing factors of trauma like high speed vehicles, stab injuries, terrorism. Major trauma does not respect and restrict itself to one organ or one system. Evaluation of patient with abdominal trauma can be most challenging task that a surgeon to be called upon to deal with. Penetrating abdominal injuries may be parietal or visceral or perforating through and through injuries. Visceral injuries may be intraperitoneal or retroperitoneal. Stomach, duodenum, small bowel, large bowel are the organs included in the study. Multiorgan injuries, exsanguinated hemorrhages, delayed presentations and the ominous reputation for high mortality and morbidity are just few of the many reasons which make this topic of penetrating injuries a fascinating one ${ }^{[1]}$.

The management of splenic injury has been subject of major re-examination over the past decade and the recognition of fatal pneumococcal septicemia in patients undergoing splenectomy has led to an interest in splenic salvage. Ultrasound, CT Scanning and Radionuclide scanning can reveal significant splenic injury but should only be pursued with an understanding of what therapeutic plan will follow if these tests are positive ${ }^{[2]}$.

The non-operative approach to the splenic injury and spleen conserving surgery has been practiced now in major trauma centers with help of CT and radionuclide scan.

The intraoperative evaluation of stomach injury includes good visualization of the esophageal hiatus, evaluation of the anterior portion of the stomach, division of gastrocolic ligament and complete visualization of the posterior aspect of the stomach. Penetrating wounds are debrided and primary closure performed. Despite the rich blood supply of the stomach, a few cases of gastric necrosis have been documented. Garfinkle reported one case of ischemic gastric necrosis along the greater curvature that he attributed to avulsion of the gastroepiploic vessels. Laceration of stomach may require gastric resection. Post-operative complications include intrabdominal abscess, particularly in the lesser- sac. Other complication is gastric fistula, needs immediate reoperation and repair ${ }^{[3]}$.

Serum amylase is sensitive but unfortunately not specific for duodenal injuries. Needle paracentesis or lavage will often be positive for blood, bile or bowel contents. But a negative duodenal lavage does not exclude all duodenal injuries. 
Duodenorrhaphy or simple repair will be successful in 70-85\% of the wounds. A single layer closure can be used vertically to avoid tension and that too is in single layer because to avoid narrowing of the lumen. Various pyloro plasties might also be included in the management of proximal duodenal injuries (Maingot. R. Abdominal operations,). Tube duodenostomy, transnasal tube through the pylorus and retro grade sump tube through a witzel tunnel jejunostomy are the various means of achieving tube decompression of the duodenum.

Epigastric pain out of proportion to the abdominal examination is often due to a retroperitoneal injury, the role of serum amylase in diagnosing pancreatic injuries is controversial. Abdominal CT scan are currently reported as having a sensitivity and specificity in excess of $80 \%{ }^{[4]}$.

ERCP is the most useful in preoperative delineation of ductal anatomy in patients with delayed presentations or missed injuries.

Although history and physical examination are valuable in the diagnosis of small bowel injury following penetrating trauma, these alone are not sufficiently accurate.

Stabogram to define peritoneal violation by injecting contrast into the stab wound tract and searching for intraperitoneal spillage radiographically and a high false-negative rate causes this procedure to be largely abandoned.

Any patient who has peritoneal signs or haemodynamically unstable proceeds promptly to exploratory laparotomy. Even in stable patients in whom there may not be intra peritoneal injury still laparotomy is indicated for the fear of retained radiolucent foreign body like cloth. In equivocal cases diagnostic peritoneal lavage which is useful in blunt injury but not in penetrating injury. Gunshot wounds present a much greater risk for significant intra- abdominal injury. Consequently all gunshot wounds traversing or in proximity to the peritoneal cavity are explored ${ }^{[5]}$.

At operation, significant bleeding will be the first priority. The small bowel should be carefully examined from the ligament of Tretiz all the way to the ileocaecal valve. Contusion of the antimesentric wall of the bowel may result in delayed perforation and seromuscular sutures can be used to imbricate the contusion into the lumen. Single holes from stab wounds or shotgun pellets car be closed without debridement. Since penetrating injuries in general occurs in pairs, careful examination of the bowel wall on the opposite side must be done to avoid missing any small perforations. If two adjacent holes are found they can be connected across the bridge of bowel and a transverse closure affected, so as not to narrow the lumen. Any large segments of bowel that are devascularized or have multiple defects, should be resected and re-anastomosed.

History is very important a high index of suspicion should be there. Flank pain, haematuria warrants evaluation regardless of the apparent location of trauma. The degree of haematuria may bear no relationship to the severity of renal injury. The great majority of renal injuries without haematuria are class III in type and the severe nature of trauma would alert the physician to possible renal injury these cases. Ground glass density in the flank suggests urinary extravasations or hematoma or preexisting mass such as hydronephrosis or tumor. Evaluation of upper urinary tract proceeds with high dose infusion pyelogram or double dose bolus pyelogram. Standard low dose intravenous urogram has a false negative rate of approximately $34 \%$ (Carlton, 1978) [5]. Infusion pyelography indentifies approximately $80 \%$ of renal injuries. IVU assess the injury and more importantly the status of the opposite kidney ${ }^{[6]}$.

A normal VU with haematuria in a trauma patient suggest minor renal contusion and rules out major renal injury. Incomplete or poor visualization of a portion of kidney suggest major renal trauma, include deep laceration, avulsion or vascular occlusion. Non visualization of a kidney on pylorography requires immediate arteriography whenever possible. CT is very useful investigation helpful in the non-operative management. The other modalities that have limited role include ROP, radionuclide scan.

Surgical exploration of all penetrating renal injuries is recommended because of the high incidence of associated intraabdominal injuries. Severe polar injuries are best treated by guillotine amputation to minimize delayed necrosis and fistula formation. Openings into the collecting systems are carefully closed with $4 / 0$ or $5 / 0$ chromic catgut. Renal capsule may be closed, if hemostasis is adequate. If further hemostasis is required, topical clotting agents such as avitene or oxycel may be applied and mattress sutures through parenchyma may be tied over bolster. Saucerised defects in parenchyma may be covered with omental or peritoneal graft. Shattered kidney requires nephrectomy to prevent hemorrhage (Peters and Bright). ${ }^{5}$ Finally flank is drained with Penrose or close suction drain. Renal vein laceration may be repaired by venorraphy. Renal arterial trauma may require a variety of repairs. Lateral arteriorrhaphy, arterial resection and repair by primary reanastomosis or autogenous repair. The role of interventional radiology includes percutaneous drainage of urinomas and infected hematomas. Embolisation is used when there is a persistent or delayed hemorrhage from a segmental vessel.

The presence or absence of haematuria is of little help. ${ }^{5}$ The ureteric injury should be suspected from the presence of extravasations in the excretory urogram it must be suspected in all abdominal gunshots. CT scan is more accurate in detecting the extravasations preoperatively. When the ureter injury is not made out during the initial exploration then it may have delayed presentations (Peter \& Bright, 1976). Retrograde pyelography gives one of the most precise preoperative study to localize the site and magnitude of the extravasations (Benson). It determines whether the lesion is complete or partial. Percutaneous antegrade technique also may be sometimes helpful when there is hydronephrosis.

In patients in whom there is not complete disruption of the ureteral continuity, passage of a double ' $\mathrm{J}$ ' ureteral stent with drainage may be all that is necessary. (Hoch et al., 1975). Uretropelvic junction disruptions and major upper ureteral injuries (greater than $2 \mathrm{~cm}$ laceration) are best treated by nephrostomy and stent, after repair with fine catgut sutures. Drainage should be provided. Lower ureteral injuries usually require tunneled reimplantation into the bladder, if this is not possible then a flap should be turned cephalad for reconstruction. When major ureteral loss is present or when it is necessary not to have any ureteral leakage postoperatively, one may ligate the ureter and perform nephrostomy either at the time or percutaneously within 24 hours, then later doing a transuretero - ureterostomy, if there is no contraindication, ${ }^{6}$ Alternatively one may choose substitution of a segment of tapered or non tapered ileum for the ureter. Nephrectomy may be the procedure of choice when there is simultaneous injury to the colon, ureter and iliac artery or aorta.

\section{Methodology}

This study consists of all penetrating abdominal injuries admitted in the trauma ward of government hospital. Once the patient is admitted the name, age, sex and mode of injury are noted. The time interval between injury and admission and time 
interval between admission and surgery are recorded. After retesting the patient and if time permits necessary investigation carried out. In those who are operated, the operative findings and methods of management are recorded. Cases are followed up till their discharge from the hospital. If death occurs the cause of death is evaluated. In those patients who died before surgery the postmortem findings are noted. The above facts are in a proforma prepared for this study.

\section{Results}

Table 1: Modes of penetrating abdominal injuries:

\begin{tabular}{|c|c|c|}
\hline Stab injury & 32 & $64 \%$ \\
\hline Bull gore & 12 & $24 \%$ \\
\hline RTA & 4 & $8 \%$ \\
\hline Others & 2 & $4 \%$ \\
\hline Total & 50 & \\
\hline
\end{tabular}

As given in the Table 1. Stab injury is the common penetrating trauma accounting $64 \%$. Therewere 12 cases of bullogre injury. 4 cases of RTA, 2 cases due to falling on iron rods in a concrete centering work site (accidental fall).

Table 2: Injury of the other organs

\begin{tabular}{|c|c|c|}
\hline Thoracic injuries & 12 & $24 \%$ \\
\hline Long bone injuries & 10 & $20 \%$ \\
\hline Head injuries & 4 & $8 \%$ \\
\hline Others & 2 & $4 \%$ \\
\hline Total & 28 & $56 \%$ \\
\hline
\end{tabular}

Table 2 shows the associated injuries in penetrating abdominal injuries. 12 patients sustained associated thoracic injuries. 10 patients had long bone fractures. 4 patients suffered from head injuries. 1 patient sustained cut throat injury. 1 patient had pelvic bone fracture. Totally 28 patients $(58 \%)$ had injuries involving other organs. This high incidence of polytrauma with penetrating injuries abdomen indicates the severity of injuries.

Table 3: The analysis of the time interval between injury - admission and admission - surgery.

\begin{tabular}{|c|c|c|}
\hline Time internal & Injury - Admission & Admission - Surgery \\
\hline$<2 \mathrm{hrs}$ & 18 & 12 \\
\hline $2-4 \mathrm{hrs}$ & 12 & 18 \\
\hline $4-6 \mathrm{hrs}$ & 8 & 8 \\
\hline $6-8 \mathrm{hrs}$ & 4 & 2 \\
\hline $8-10 \mathrm{hrs}$ & 3 & 1 \\
\hline $10-12 \mathrm{hrs}$ & 3 & 4 \\
\hline$>12 \mathrm{hrs}$ & 2 & 5 \\
\hline
\end{tabular}

Table 3: shows 38 cases took less than 6 hrs from time of injury to admission. The fastest to arrive was within 30 minutes from the injury.

The average time duration between admission and surgery was 4 hrs.

\section{Discussion}

\section{Liver Injuries}

There were totally 8 cases of liver injury, in this 6 cases were due to stab injury and 2 cases were due to bull gore. The commonest cause of penetrating liver injury in Ben Taub General hospital, Hauston was Gunshot wounds accounting for $50.60 \%$ and stab injuries accounting for $33.90 \%$. The incidence of associated organ injuries is a significant factor in patients sustaining liver injures. In this study only 3 cases were isolated liver injuries and remaining were associated with other organ injuries. The different ways in which the 8 cases of liver injuries were managed as follows, application of Gel foam and suture hepatorraphy was done in 5 cases. In 1 case there was no active bleeding hence no repair done. In 1 case shattered liver present so segmental hepatectomy done. In 1 case omental pack was kept in deep lobar laceration to control bleeding. Peritoneal lavage with normal saline and open drainage was kept in all cases.

In this study 2 out of 8 cases died giving morality rate of $25 \%$. Mortality in our case was due to the severe associated injuries and one died of septicemia at the end of the $4^{\text {th }}$ post operative day. Two cases developed subphrenic abscess.

The Mortality rate at the Ben Taub General hospital in Houston from 1979 to 1984 was $10-15 \%$. The incidence of post operative perihepatic abscess range from $3.5 \%$ to $22 \%$. Post operative perihepatic abscess was diagnosed clinically in the patients who remained continuously febrile after 5-7 days and had persistent leucocytosis and foul smelling drainage from open drainage. All the patients were treated conservatively. One patient develops pneumonia with hyperpyrexia and it was confirmed by x-ray chest and treated conservatively ${ }^{[7]}$. In this study no complication of biliary leak.

\section{Splenic Injuries}

There were totally 4 cases of splenic injury. Of these 4 cases 3 cases were due to stab injury and 1 case was due to bull gore injury, where as the series form the Ben Taub General hospital in Houston has reported an incidence of gunshot splenic injury as $7.6 \%$ and stab injuries as $7 \%$ among penetrating splenic injuries ${ }^{[8]}$.

In this study only one case had isolated splenic injuries. 1 case was associated with diaphragmatic injuries and 1 case had associated pancreatic injury with retroperitoneal hematoma. 1 case was associated with stomach and small bowel injury.

In this study all the injured spleen have undergone splenectomy. Whereas the series from Ben Taub Hospital, Houston report 45$50 \%$ of injured spleen have undergone repair instead of splenectomy that too splenorrhaphy was accompanied in $51 \%$ of patients with penetrating mechanism of injury.

The grading of the splenic injury has a significant impact on treatment. In our study out 4 patients, 3 patients were haemodynamically unstable and had associated intra abdominal injuries, so we could not perform splenorrhaphy for these patients.

In this study two cases died in the immediate post operative period due to hypovolumic shock and multiple organ failure. 2 patients had fever ranging from $99^{\circ} \mathrm{F}$ to $102^{\circ} \mathrm{F}$ upto $4^{\text {th }}$ post operative day and 1 patient had consolidation of the left lower lobe. All of them are treated conservatively with antibiotics and antipyretics. Three patients had wound infection.

\section{Stomach Injuries}

There were totally 8 cases of stomach injuries. In this 6 cases were due to stab injuries, 1 case due to Bull gore and 1 case due to RTA. Where as the series from Ben Taub Hospital report an incidence of Gun shot stomach injuries as $17.3 \%$ and stab stomach injuries as $12.6 \%{ }^{[9]}$.

In this study isolated stomach injury was present in 3 cases. All remaining cases were associated with other organ injuries, of which retroperitoneal haematoma was present in 3 cases, liver injury in 2 cases, splenic injury in 1 case, duodenal injury in 1 case, diaphragmatic and small bowel injury in 1 case. Pre operatively all cases were confirmed by the passage of bright red 
blood through Ryle's tube and the presence of free air in X-Ray abdomen. In this study in all stomach injuries, the entrance and exit sites of the penetrating wound were visualized. Then the stomach was closed in two layers utilizing an inner continuous suture with 2-0 vicryl (or) catgut in full thickness manner. This layer is then imbricated with sero muscular layer of interrupted Lambert sutures using $2-0$ or $3-0$ silk. In one patient after gastrorraphy AGJ and JJ was done and that patient died of septicemia on $4^{\text {th }}$ post operative day. One more patient died of multiorgan failure due to concomitant liver and small bowel injuries. One patient developed consolidation of left lower lobe with left subphrenic abscess and three patients had wound infection and all by them treated conservatively. In all cases peritoneal lavage done with normal saline and open drainage kept.

\section{Duodenal injuries}

There were totally 4 cases of duodenal injuries of which 3 cases due to stab injury and 1 case due to bull gore. According to six recent series the incidence of penetrating duodenal injury was $85 \%$ and blunt duodenal trauma was $15 \%$. All patients were taken up for exploratory laporotomy. All patients underwent duodenorrhaphy in 2 layers. 2 patients underwent gastrojejunostomy, in 2 patients a serosal jejunal patch was kept. One patient tube decompression of duodenum was done. John A Weigelt also states that $80 \%$ of patients with duodenal injuries require simple duodenorrhaphy ${ }^{[10]}$.

In this study one case died $5^{\text {th }}$ post-operative day due to septicemia and concomitant liver and colonic injuries. But this may not reflect the real problem because the number of cases studied was too small and the period of study was also short. Levison et al. 1984 in a recent series reports a mortality of $16.7 \%$ for blunt duodenal trauma and mortality of $7.5 \%$ for penetrating duodenal trauma.

Incidence of duodenal fistula in our study was $15 \%$.

\section{Pancreatic Injuries}

There were totally 2 cases of pancreatic injuries. Of which one case was due to stab injury and one case was due to bull gore injury. Gregory.J. Jurkovich states that penetrating trauma accounts for two third and blunt trauma accounts for one third of pancreatic injuries. In our study $95 \%$ of the patients had associated organ injuries which are in accordance with the reports of Balasegaram.M. 1979, Graham. J M. et al. 1978, Smego.DR et al. 1985, and Stone.HH et al. $1981^{[6]}$.

One patient was taken up for cystojejunostomy as he presented with pseudocyst of pancreas. In one patient who had multiple stab injury of the bowel with pancreatic injury, had surgery done outside and was referred to our center with enterocutaneous and pancreatic fistula.

In this study the mortality rate for pancreatic injury was $50 \%$.The combined mortality from several large series of pancreatic trauma patients range from 10- 25\%. Our morbidity rate was $70 \%$. In this study the incidence of pancreatic fistula was $50 \%$ which healed with conservative management.

\section{Jejunum and Ileum Injuries}

There were totally 10 cases of small bowel injuries. Of which 7 cases were due to stab injury and 2 cases were due to bull gore injury and 1 case was due to accidental fall injury. Whereas the incidence of small intestinal injury following penetrating trauma exceeds $80 \%$ with gunshot wound and $30 \%$ with stab injuries that penetrate the peritoneum.
In this study only 3 cases had isolated small bowel injury. In the remaining 4 cases had associated mesenteric tears, one case was associated with colonic injuries and other two cases were associated with liver, spleen and stomach injuries separately.

After laparotomy, thorough search for wounds from the ligament of Treitz to the ileocaecal valve was done in all small bowel injuries. In this study, three cases of hematomas and serosal lacerations of small bowel were 'Turned In' using Lembert sutures placed in a transverse fashion. In 3 cases with questionable viability of bowel, we have done resection and anastomosis in 2 layer transversely using inner continuous $2 / 0$ vicryl and outer lembert sutures with $2 / 0$ silk. In all cases thorough peritoneal irrigation with saline done and open drainage was kept.

In this study one case died due to concomitant colonic injury, because the number of cases studied was too small this may not reflect the real problem. In our study two patients had wound infection and two had intrabdominal abscess, both of these were treated with conservative management.

\section{Caecum and ascending colon injury}

There was only one isolated case of caecal injury due to stab injury. There was laceration of caecum and mild hematoma in mesentry, which was sutured in 2 layers using 2-0 vicryl and 2-0 silk. Thorough laparotomy done and peritoneal irrigation with saline done and open drainage kept.

Post-operative period was uneventful, and patient recovered well. On $6^{\text {th }}$ day there was wound infection, which was treated conservatively.

\section{Transverse colon injuries}

There were totally 3 cases of transverse colon injuries. 2 cases were due to stab injury and 2 cases due to bull gore injury. 2 cases were small laceration of transverse colon and hematoma in mesocolon which was repaired by lembert sutured transversely with 2-0 silk. One patient had transverse colon transaction and mesocolon rent, which was repaired after trimming the edges and confirming the viability, anastomosis done in 2 layers using 2-0 vicryl and 2-0 silk. Peritoneal lavage done with normal saline and open drainage kept.

In this study all three cases were recovered better and 1 patient had wound infection, which was treated with conservative management.

\section{Descending and Sigmoid Colon Injuries}

There were totally 3 cases of injury to the sigmoid colon. 2 cases due to stab injury and 1 case due to bull gore injury. The bull gore injury case had associate injury to the scrotum. 2 cases had perforation of the sigmoid colon with sigmoid mesocolon rent. Both the cases were treated with primary 2 layer closure with 20 silk. In $3^{\text {rd }}$ case there was laceration of sigmoid and ascending colon, in this resection and anastomosis done in 2 layer using 20 vicryl and 2-0 silk. Proximal covering transverse colostomy done, thorough peritoneal lavage given with normal saline and flank drain kept.

In all 3 cases postoperative period was uneventful. One case had wound infection at $5^{\text {th }}$ postoperative day, which was treated conservatively.

\section{Retroperitoneal hematoma}

There were 3 cases of mild retroperitoneal hematoma which were associated with other injuries. Nothing specific was done for these hematomas. All patients had uneventful recovery. 


\section{Diaphragmatic injuries}

There were totally 3 cases of diaphragmatic injuries. Of which 2 cases were due to stab injury and one case was due to bull gore injury. All cases were associated with intrabdominal injuries of which, two cases were associated with splenic injuries and one case was associated with combined stomach and splenic injuries. In this study, after laparotomy only in one case we had converted into thoracoabdominal incision and in remaining cases repair was done through abdominal approach only. The rent was closed with simple sutures with 1- prolene or with other non absorbable suture materials or with figure of eight sutures of the same material. In all cases ICD was done after closure. One patient died on $4^{\text {th }}$ postoperative day due to septicemia.

\section{Negative celiotomies}

In this study there were 4 cases of negative celiotomies. Where as in Feliciano et al. 1984, Shorr et al. 1988, series, the negative celiotomies were from $5.8 \%$ to $7.4 \%$. In this study after confirmation of peritoneal penetration by wound exploration, exploratory laparotomy was done in all cases. There was no viscous or vascular injury, and there was no missed injury in our study. All were discharged after an uneventful post operative period.

\section{References}

1. Peters PC, Bright TO Ill. Blunt Renal Injuries Uro Clinic North America. 1977; 4:17.

2. Sagalowsky I, Paul Peters0. Genito urinary Trauma. Campbell's urology.

3. Snyder WH Ill et al. The Surgical Management of duodenal trauma, Arch Surg. 1980; 115:422-429.

4. Ivaturary RR, Nallathambi M, Gaudino J et al. Penetrating duodenal injures Analysis of 100 consecutive cases, ann Surg. 1985; 202(2):153-158.

5. Jones RC. Management of Pancreatic trauma: ann Surg. 1978; 187(5) 555-564.

6. Carlton CE Jr. Injuries of the kidney and ureter. In Harrison, JH et at (Eds) Campbell's urology. 4th Edition, 1978, 1.

7. Flint LM, McCoy M, Richardson JD et al. Duodenal injury: Analysis of common mis conceptions in diagnosis and treatment Ann Surg. 1980; 191(6): 697- 702.

8. Traub A, Giebink GS, Smith $\mathrm{C}$ et al. Splenic recticuloendothelial function after splenectomy, splenic repair and splenic auto transplantation. N Eng J med. 1987; 317:1559.

9. Levison MA, Peterson SR, Sheldon GF et al. Duodenal trauma experience of a trauma center. J trauma. 1984; 24(6):475-480.

10. Moaninch JW, Carrol PR. Renal trauma, Kidney preservation through improved vascular control-A refined approach. J trauma. 1982; 22:285. 